\title{
Flare parameters inferred from a 3D loop model data base
}

\author{
Valente A. Cuambe, ${ }^{1,2 \star}$ J. E. R. Costa ${ }^{1 \star}$ and P. J. A. Simões ${ }^{3}$ \\ ${ }^{1}$ Astrophysics Division, National Institute for Space Research (INPE), Av. Dos Astronautas, 1758 São Jose dos Campos, SP, Brazil \\ ${ }^{2}$ Physics Division, Eduardo Mondlane University (UEM), Av. Julius Nyerere 1569, Maputo, Mozambique \\ ${ }^{3}$ SUPA School of Physics and Astronomy, University of Glasgow, Glasgow G12 8QQ, UK
}

Accepted 2018 March 29. Received 2018 March 29; in original form 2017 July 26

\begin{abstract}
We developed a data base of pre-calculated flare images and spectra exploring a set of parameters which describe the physical characteristics of coronal loops and accelerated electron distribution. Due to the large number of parameters involved in describing the geometry and the flaring atmosphere in the model used, we built a large data base of models $(\sim 250000)$ to facilitate the flare analysis. The geometry and characteristics of non-thermal electrons are defined on a discrete grid with spatial resolution greater than 4 arcsec. The data base was constructed based on general properties of known solar flares and convolved with instrumental resolution to replicate the observations from the Nobeyama radio polarimeter spectra and Nobeyama radioheliograph (NoRH) brightness maps. Observed spectra and brightness distribution maps are easily compared with the modelled spectra and images in the data base, indicating a possible range of solutions. The parameter search efficiency in this finite data base is discussed. 8 out of 10 parameters analysed for 1000 simulated flare searches were recovered with a relative error of less than 20 per cent on average. In addition, from the analysis of the observed correlation between NoRH flare sizes and intensities at $17 \mathrm{GHz}$, some statistical properties were derived. From these statistics, the energy spectral index was found to be $\delta \sim 3$, with non-thermal electron densities showing a peak distribution $\lesssim 10^{7} \mathrm{~cm}^{-3}$, and $B_{\text {photosphere }}$ $\gtrsim 2000 \mathrm{G}$. Some bias for larger loops with heights as great as $\sim 2.6 \times 10^{9} \mathrm{~cm}$, and looptop events were noted. An excellent match of the spectrum and the brightness distribution at 17 and $34 \mathrm{GHz}$ of the 2002 May 31 flare is presented as well.
\end{abstract}

Key words: Sun: activity -Sun: flares - Sun: magnetic field -Sun: radio radiation.

\section{INTRODUCTION}

A solar flare is one of the most intense energetic phenomena in the solar atmosphere. It manifests itself as a sudden, rapid enhancement of intensity in almost all electromagnetic wavelengths (Fletcher et al. 2011). It is known that these phenomena can accelerate electrons up to hundreds of $\mathrm{MeV}$. The excess of energy in tens of seconds is of the order of $10^{32}$ ergs (see reviews by Bastian, Benz \& Gary 1998; Fletcher et al. 2011; White et al. 2011). The process occurs in a magnetic loop or arcades over active regions as observed at ultraviolet wavelengths (e.g. Liu et al. 2015).

The accelerated electrons moving along magnetic field lines can be trapped due to magnetic mirroring, thus producing radio emission via the gyrosynchrotron mechanism in a trapped volume. During this process, a fraction of these electrons precipitates into the chromosphere, where they produce hard X-rays via the bremsstrahlung process (e.g. Melrose \& Brown 1976).
The characteristics of this radio emission (spectrum, polarization and spatial distribution) are strongly dependent on the magnetic field strength and geometry, as well as the properties of the non-thermal electrons such as energy, pitch angle and spatial distribution (Alissandrakis \& Preka-Papadema 1984; Klein 1987; Melnikov et al. 2002; Simões \& Costa 2006, 2010; Fleishman, Nita \& Gary 2011; Costa et al. 2013).

A great variety of source morphologies of a flare can be widely observed when X-ray and radio observations are combined. These source morphologies can be interpreted as magnetic arcades, as an interaction between two or more loops, as well as in other ways. In microwaves, it is common to use images from the Nobeyama radioheliograph (NoRH) (Nakajima et al. 1994) at fixed frequencies of 17 and $34 \mathrm{GHz}$ with spatial resolution greater than 10 arcsec, and data from the Very Large Array (VLA) and the Owens Valley Solar Array (OVSA; Hurford et al. 1984). Thus, we used the largest available archive of microwave flare images from Nobeyama to constrain our parameter set in the definition of the data base of models. In the X-ray band, we use the Reuven Ramaty High Energy

^E-mail: valente.cuambe@inpe.br (VAC); joaquim.costa@inpe.br (JERC) 
Solar Spectroscopic Imager (RHESSI) combining both images with a spatial resolution of 2.3 arcsec and spectra.

The lack of knowledge of the detailed coronal magnetic field usually leads to the creation of a simple homogeneous source model to analyse the emission. This results in incorrect inferred parameters according to Costa et al. (2013). On the other hand, a 2D model of the dipolar magnetic field geometry was proposed to replicate the observed microwave emission for specific flares (e.g. Petrosian 1981; Alissandrakis \& Preka-Papadema 1984; Nindos et al. 2000; Kundu et al. 2001).

To correctly describe a spatially varying flare source, it is necessary to define the strength and geometry of the magnetic field, spatial distribution of the non-thermal electrons, and, to a lesser extent, the microwave emission, the thermal plasma density and temperature, which can be relevant to the free-free absorption and Razin effect.

Costa et al. (2013; hereafter PAPER1) developed an inhomogeneous model using 3D dipolar loop geometry, and a limited number of parameters, to describe the observations. In line with PAPER1, we built a data base with simplified 3D loop geometry and a distribution of parameters. We calculated over 250000 models. In this data base, we inserted two new parameters to improve the description of the geometry of the magnetic dipole model.

Although we understand the theory of gyrosynchrotron emission, we require further information on the geometry and strength of the magnetic field in the corona, $B(x, y, z)$, and the distribution of the non-thermal electrons in space and energy to construct the flare scenario. All the above information is important to compute a model to explain a flare. It is very complicated to select a set of parameters with confidence that they exist in the solar atmosphere and reflect the observations. Consequently, both the process of parameter selection and the calculations are time consuming in practice, as reported in the literature (Alissandrakis \& Preka-Papadema 1984; kucera et al. 1993; Lee, Gary \& Zirin 1994; Bastian, Benz \& Gary 1998; Simões \& Costa 2006; Fleishman, Nita \& Gary 2009; Simões \& Costa 2010; Costa et al. 2013; Kuznetsov \& Kontar 2015; Nita et al. 2015).

Past and current models cannot constrain the observations well, not even the simplest, uniform source model. This makes the parameter space for forward-fitting modelling huge. Thus, the proposed large pre-calculated data base of 3D simplified models results in a faster search of the parameters in a flare analysis. Observed spectra and brightness distribution maps can be easily compared with the modelled spectra and images in the data base, indicating a possible range of solutions.

In this paper, we characterize a solar flare based on key parameters of its microwave emission in a simple $3 \mathrm{D}$ geometry of a magnetic loop. Because of the high number of parameters involved, we offer a large data base of pre-calculated model spectra and images to better explore the parameter range that explains the observations. We also aim to obtain statistical flare properties inferred from our data base solutions constrained by Nobeyama radio polarimeter (NoRP) and NORH observations.

\section{MODEL DESCRIPTION}

We calculated the gyrosynchrotron emission from non-thermal electrons in a 3D magnetic loop. The loop is modelled as a dipole magnetic field. The magnetic induction of any voxel (volume unit) in space at position $\mathbf{r}$, from the dipole magnetic momentum $(\mu)$, is

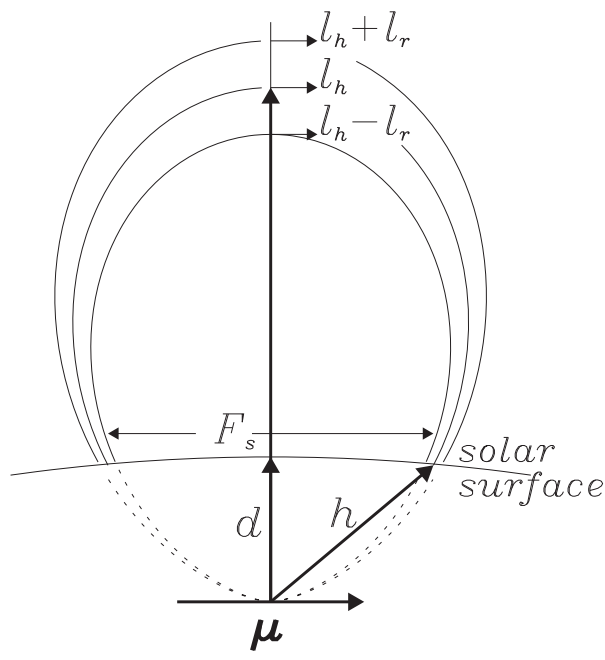

Figure 1. Magnetic dipole model of PAPER1.

calculated by

$B=\frac{3(\mu \mathbf{r}) \mathbf{r}-\mu \mathbf{r}^{2}}{\mathbf{r}^{5}}$.

The absolute value of the magnetic momentum was adjusted to give the desired magnetic field to the loop foot-point. The dipole is placed below the solar surface at a depth $d$ as shown in Fig. 1 . The maximum magnetic field strength is a free parameter for the lowest level of the loop volume. The flaring volume was constructed around the central field line with a circular cross-section with radius at the apex $\left(l_{\mathrm{r}}\right)$. Thus, the geometrical free parameters are the loop height $\left(H_{\mathrm{arc}}=l_{\mathrm{h}}-d\right.$, where $l_{\mathrm{h}}$ is the distance from the dipole momentum to the central field line at the loop apex), feet separation $\left(F_{\text {sep }}\right)$ and apex radius. The dipole depth $d$ below the surface was calculated from the selected loop height and feet separation which in turn changed the mirror ratio.

The electron distribution is defined by its energy distribution in the form of a power law (with power index $\delta$ as a free parameter) from $10 \mathrm{keV}$ to $100 \mathrm{MeV}$, in a homogeneous pitch angle distribution. The spatial distribution of non-thermal electrons was set to be symmetrical in relation to the loop apex. No radial variation is included in our calculations.

Fig. 2 shows the empirical modified functions in relation to PAPER1 that concentrate the electrons at loop-top or foot-point and homogeneous distribution through a control parameter called $\epsilon$. We defined $\epsilon$ as the selection parameter for the distributions used in the model; it varies from high concentration at the foot-point (with low concentration at the loop-top) to low concentration at the foot-point (with high concentration at the loop-top) passing through the homogeneous distribution. In this current version, we used intervals to name the non-thermal electron distributions at loop-top $(1.5 \leq \epsilon$ $\leq 2.0)$, foot-point $(0 \leq \epsilon \leq 0.6)$, and homogeneous $(0.6<\epsilon<1.5)$.

Although the model includes bremsstrahlung radiation from the ambient thermal plasma, this was not included in our calculations for this data base. Therefore, the ambient density was defined as an exponential decay from $10^{13}$ to $10^{8} \mathrm{~cm}^{-3}$. Thus, for a given set of parameter reference values, the gyrosynchrotron emission is calculated according to Ramaty (1969), using a code by Simões \& Costa (2006, 2010), Simões (2009) and Costa et al. (2013) to obtain the emission and self-absorption coefficients and the $3 \mathrm{D}$ radiative transfer. 


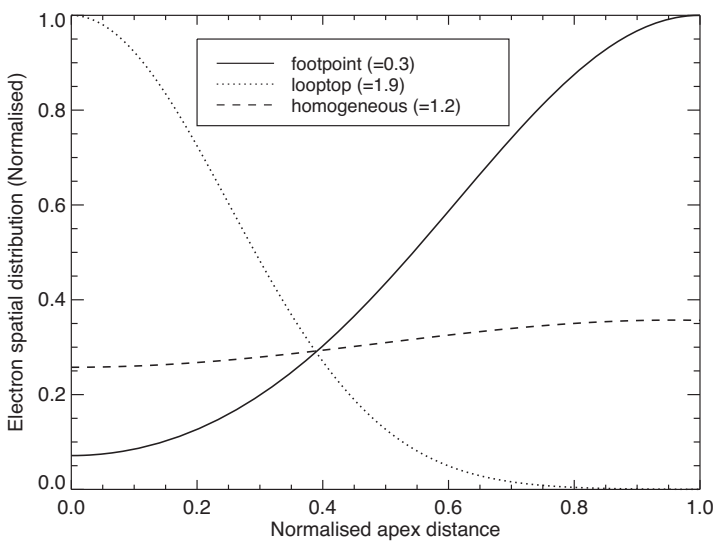

Figure 2. This figure shows the non-thermal electron spatial distribution at a normalized distance from the loop-top $(x$-axis $=0)$ to the foot-point $(x$-axis $=1.0)$.

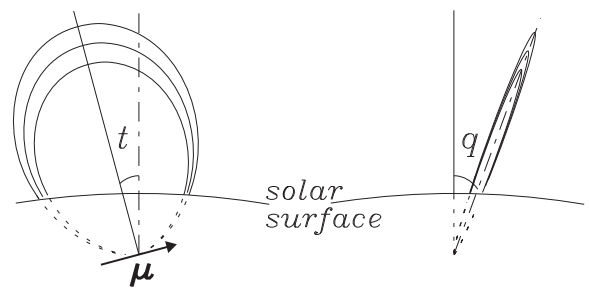

Figure 3. Magnetic dipole model. The left-hand panel shows the asymmetry (loop variation along dipole plane); the right-hand panel shows the loop inclination. The loop was rotated in relation to the vertical line for a side view and inclined by $q$ (the angle between the vertical continuous line and the inclined dot-dashed line) angle.

Moreover, two further degrees of freedom were added to the dipole magnetic field relative to the geometry of PAPER1 to complete the proposed model: the loop inclination and loop asymmetry. These two geometric parameters represent the variations in the direction of the dipole axis. The left-hand panel of Fig. 3 shows the loop asymmetry (angle $t$ ) which refers to the variation along the dipole plane in the magnetic loop (i.e. in Fig. 1, when the dipole axis tilts to the right/left it changes the foot-point depth accordingly). On the other hand, the loop inclination is the vertical variation of the plane that contains the loop shown by the angle $q$ on the righthand panel of the Fig. 3 (i.e. the plane that contains the loop tilts forwards/backwards in Fig. 1).

A notable improvement from the asymmetry parameter comes from the visual interpretation of the observational data. Changes in this parameter result in a different magnetic field for each foot-point. From the magnetogram, it can be clearly seen that the magnetic field is not symmetrical in both legs of the loops in the majority of the active regions. In this current version of the model, changes in this parameter result in slightly different distributions of the magnetic field in the loop and consequently changes in the brightness distribution. On the other hand, NoRH images sometimes show apparently inclined loops that were not included in PAPER1. Changes in this parameter have the effect of significantly altering the viewing angles.

The flare geometry was defined inside a cube with $16 \times 16 \times 16$ voxels. For a typical flare volume, this is equivalent to a spatial resolution of about 2 arcsec.

\section{MODEL DATA BASE}

Modelling the flaring region can be a typical inverse problem starting with the observables, in this case the flare images and spectra, to reverse the inherent physical equations to infer the flare scenario. On the other hand, the direct (forward) approach may also be used assuming some plasma configuration attempting to match some observation. In terms of models, the observables result from the solution of a set of integral equations which in practical terms cannot be inverted. Therefore, instead of an inverse problem, a forward method was used here to explore a large set of parameters (possible flare conditions) and their resultant calculated images and spectra (our 3D model's data base). Finally, to be used to compare with real observations.

The data base was built to match the four NoRP bands, namely 3.7, 9.4, 17, and $34 \mathrm{GHz}$. These frequencies are ideal to study the gyrosynchrotron spectrum without contamination from lowfrequency plasma emission mechanisms in most of the known flares (Nita, Gary \& Lee 2004).

Each model in the data base is constructed with 17 parameters the number of voxels in each cube, heliographic position, azimuth, control parameter $\epsilon$, ambient density, non-thermal electrons number density, loop radius, feet separation, loop height, loop asymmetry, loop inclination, temperature, energy, spectral index, pitch angle, viewing angle, and magnetic field strength. Fifteen of these parameters have been previously described in PAPER1, and two more have been implemented in this paper as mentioned earlier, the loop inclination and loop asymmetry (see Fig. 3). In this current version, the number of voxels was reduced to $16 \times 16 \times 16$. This reduction in the number of voxels speeds up the calculations of models without degrading the space resolution in relation to all the available instruments too much.

The range of the energy spectral index was set following the event list of flares observed with the NoRP from 1992 to 2014 . From this period, we selected 233 flares that had a peak frequency lower than 9.4 GHz. From this sample, we were able to use $17-$ and $34-\mathrm{GHz}$ bands to calculate the radio spectral index in the optically thin part of the spectrum, as

$\alpha=-\frac{\log \left(F_{34} / F_{17}\right)}{\log (34 / 17)}$.

The emission spectral index $(\alpha)$ derived from the fluxes at 17 and $34 \mathrm{GHz}$ is related to the spectral index of emitting electrons for the homogeneous source ( $\alpha=0.90 \delta-1.22$ ) according to Dulk $\&$ Marsh (1982). Then the power index of the energy distribution was fitted assuming a Gaussian distribution $(\bar{\delta}=2.7, \sigma=0.8)$ with 95.44 per cent confidence. This range was only used to determine the energy spectral index $(\bar{\delta} \pm \sigma)$ to construct the model data base. Although the model is implemented with a multipower-law index, we constructed the data base with single power law to minimize the number of parameters, as a first approach.

From the literature (e.g. Gary 1985; Lee et al. 1994, 2009), we found that the instantaneous number of non-thermal electrons at the time of the burst maximum are in the range $1 \times 10^{33}-1 \times 10^{42}$. Within this range, the non-thermal electron number density can be calculated dividing the total number of non-thermal electrons by the loop volume. However, this range of non-thermal electrons was later fine-tuned in the data base using the observed range of measured flux densities.

Therefore, the non-thermal electron number density, the powerlaw index of the energy distribution $(\delta)$, the reference magnetic field at the photosphere $(B)$, the loop asymmetry, loop azimuth, loop apex 
Table 1. The range of parameters in the data base.

\begin{tabular}{lllll}
\hline No. & Parameter & Min & Max & Symbol/unit \\
\hline 1 & Electron fill & 0.2 & 2 & $\epsilon$ \\
2 & Spectral index & 1.0 & 4.4 & $\delta$ \\
3 & Electron density & $1 \times 10^{6}$ & $1 \times 10^{8}$ & $N_{\text {nth }} / \mathrm{cm}^{-3}$ \\
4 & Asymmetry & -50 & 50 & $\mathrm{q} / \mathrm{per} \mathrm{cent}$ \\
5 & Azimuth & -90 & 90 & $\mathrm{Az} / \mathrm{Deg}$ \\
6 & Apex radii & 0.002 & 0.02 & $R_{\text {arc }} / \mathrm{R}_{\odot}$ \\
7 & Foot separation & 0.008 & 0.06 & $F_{\text {sep }} / \mathrm{R}_{\odot}$ \\
8 & Loop height & 0.008 & 0.06 & $H_{\text {arc }} / \mathrm{R}_{\odot}$ \\
9 & Loop inclination & -40 & 40 & $t / \mathrm{deg}$ \\
10 & Magnetic field & 800 & 3500 & $\mathrm{~B} / \mathrm{G}$ \\
\hline
\end{tabular}

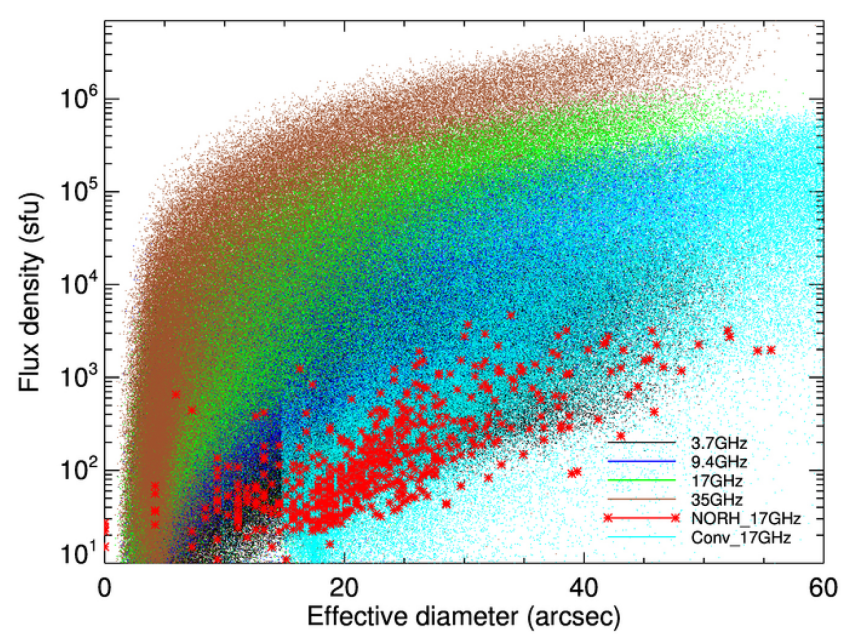

Figure 4. Effective diameter versus flux density in the data base in four frequency bands without convolution. The red asterisks are the selected NoRH data obtained from the NoRH list at $17 \mathrm{GHz}$, and cyan dots are the $17 \mathrm{GHz}$ convolved models (beam 10 arcsec).

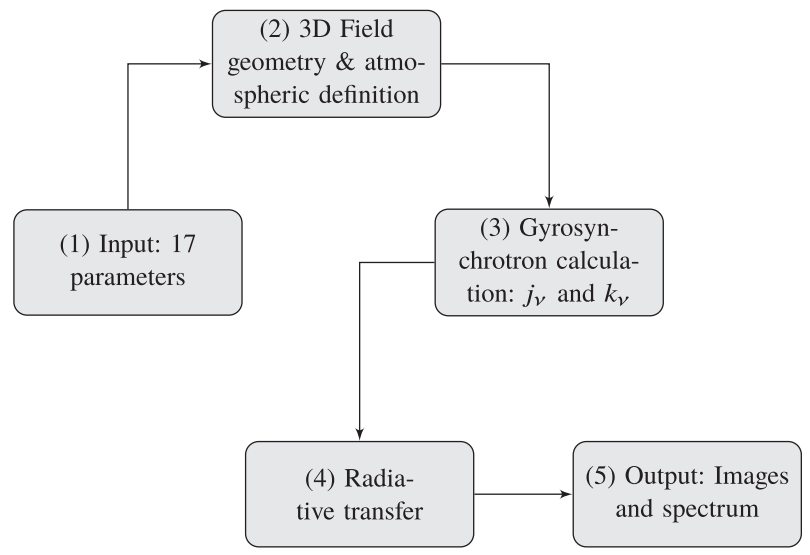

Figure 5. The block diagram that shows the logical scheme to compute a model in the data base.

radius, foot separation, and loop height and loop inclination were varied in the ranges shown in Table 1 . The energy range of the power-law distribution of non-thermal electrons - from $10 \mathrm{keV}$ to $100 \mathrm{MeV}-$ in a homogeneous pitch angle distribution was adopted as well. The parameter limits in Table 1 were randomly chosen within the selected ranges as the data base was expanding to reflect the range of flux densities observed by NoRP.
In Fig. 4, we show the effective diameter versus flux density for each magnetic loop model in the data base with randomly distributed parameters calculated in the four above-mentioned frequency bands labelled with different colours. The red asterisks are the Nobeyama flares obtained from the NoRH website flare list. ${ }^{1}$ The effective diameter is derived from the square root of the source emitting area. It is noted that the flux densities at $17 \mathrm{GHz}$ are overestimated (see the difference between cyan dots and red asterisks in Fig. 4) compared with observations. Many models were kept in the data base because they physically reflect reasonable flare parameters.

In PAPER1, it was decided to keep total volume constant to decrease the parameter space. However, considering the results of the NoRH flares at $17 \mathrm{GHz}$ in this current study, the geometric parameters were randomized to explain observed flare geometry characteristics (sizes). It became clear that the data base had been inflated with a combination of parameters that did not reflect the observations of $17 \mathrm{GHz}$ flares. This constitutes an important result that will be analysed in Section 6.1.

\section{DATA BASE SEARCHING MECHANISM}

A given observed NoRP spectrum and NoRH images (not restricted to), both at 17 and $34 \mathrm{GHz}$ if available, can be compared with all spectra and images in the data base using a classical fitting:

$\chi^{2}=\sum_{i} \frac{1}{\psi_{\mathrm{Obs}_{\mathrm{i}}}^{2}}\left(\psi_{\mathrm{Mod}_{\mathrm{i}}}-\psi_{\mathrm{Obs}_{\mathrm{i}}}\right)^{2}$,

where $\psi_{\text {Obs }}$ is any measured observable, and $\psi_{\text {Mod }}$ is the same as that determined from the model in the data base. The sum is performed over all observable $i$. The output of this search is a sequence of the best models in the data base ordered by increasing $\chi^{2}$.

This search is carried out by feeding (1) four flux densities in $\mathrm{sfu}^{2}$ $\left(F_{v}\right.$, where $v$ is $3.7,9.4,17$, and $34 \mathrm{GHz}$ ) observed or inferred from observations in other frequency bands, (2) the heliographic position of the burst (active region), and (3) the images if available from both 17 and $34 \mathrm{GHz}$. If the images are unavailable, the user is invited to input the estimated azimuth obtained from the magnetogram to improve the chance of obtaining good results.

\section{FLARE SEARCH IN THE DATA BASE}

We emphasize that each model in the data base is performed following the steps previously described in PAPER1, and briefly explained

\footnotetext{
${ }^{1}$ http://solar.nro.nao.ac.jp/norh/html/event/

${ }^{2}$ Solar flux unit $\left(1 \mathrm{sfu}=10^{-19} \mathrm{erg} \mathrm{s}^{-1} \mathrm{~cm}^{-2} \mathrm{~Hz}^{-1}\right)$.
} 
Table 2. Parameters of the simulations and respective solutions.

\begin{tabular}{|c|c|c|c|c|c|c|c|c|c|c|}
\hline Parameter & $\begin{array}{c}\epsilon \\
(1)\end{array}$ & $\begin{array}{c}\delta \\
(2)\end{array}$ & $\begin{array}{c}N_{\text {nth }}(\log ) \\
\text { (3) }\end{array}$ & $\begin{array}{c}q \\
(4)\end{array}$ & $\begin{array}{l}\mathrm{Az} \\
\text { (5) }\end{array}$ & $\begin{array}{l}R_{\text {Arc }} \\
\text { (6) }\end{array}$ & $\begin{array}{l}F_{\text {sep }} \\
(7)\end{array}$ & $\begin{array}{c}H_{\text {Arc }} \\
(8)\end{array}$ & $\begin{array}{c}t \\
(9)\end{array}$ & $\begin{array}{c}B \\
(10)\end{array}$ \\
\hline A & 1.37730 & 2.42539 & 6.38534 & 16.5031 & 75.6650 & 0.0125987 & 0.0241344 & 0.0502481 & 36.3673 & 1505.44 \\
\hline Mean & 1.04766 & 2.44599 & 6.51370 & -11.6934 & 74.7544 & 0.0100789 & 0.0247609 & 0.0446416 & 18.2583 & 1450.04 \\
\hline B & 0.457685 & 1.79625 & 7.19426 & -13.1240 & -64.3377 & 0.00675105 & 0.0365211 & 0.0286779 & -16.8787 & 1601.53 \\
\hline Mean & 1.13904 & 2.26227 & 7.14912 & -0.656433 & -70.1029 & 0.0118904 & 0.0356033 & 0.0349081 & 0.0682524 & 2102.58 \\
\hline
\end{tabular}

in Section 2. The logical scheme is summarized in the block diagram shown in Fig. 5. There are five main steps: (1) The input parameters are randomized, (2) the flare atmosphere and the geometry of magnetic field $B(x, y, z)$ are defined, (3) the gyrosynchrotron coefficients are calculated following Ramaty (1969) using a code by Costa et al. (2013), (4) the radiative transfer is performed, and finally (5) the output are four images and spectrum of each model. It is important to note that all physical parameters in each data cube can also be recovered from the data base.

To test the functionality of this data base, we simulated the search of one model by extracting it randomly from the model data base and then sought the nearest match. The idea is to compare its brightness map and spectrum with the other elements in the data base to look for the best match and compare both sets of parameters. To statistically determine the quality of the search this process was repeated 1000 times. To clarify our intent, let us name the model picked at random from the data base 'simulation' and the best match found in data base 'solution'.

To quantify the result, we defined a variable $\zeta$ to reflect the similarity between the parameters of the models in the data base and the simulated one, as follows:

$\zeta_{i}=\frac{\left|\operatorname{Par}_{i}^{\mathrm{Sim}}-\mathrm{Par}_{i}^{\mathrm{Mod}}\right|}{\operatorname{Par}_{i}^{\mathrm{Sim}}}$.

In equation (4) above, the $\operatorname{Par}_{i}^{\mathrm{Sim}}$ defines each parameter used in the simulations described in the second column of Table 1, and $\operatorname{Par}_{i}^{\mathrm{Mod}}$ is the same as the previous, but for the tested model found in the data base (solution), $i=1,2, \ldots, 10$.

The similarity $\zeta$ is the summation of relative error of all 10 parameters used to verify the solution. It is important to note that we chose only 10 parameters that cannot be obtained directly from observations. We also note that if the parameters are measured in units of angles (e.g. azimuth and inclination) the denominator of equation (4) is set to $90 \mathrm{deg}$ to better reflect the errors due to misalignment.

In order to choose the best solution, two different methods were used: (i) We chose the model with the lowest $\chi^{2}$ in the data base, (ii) we calculated the weighted mean of a hundred models ordered by increasing $\chi^{2}\left(\overline{\operatorname{Par}}_{i}{ }^{\text {Mod }}\right)$. The weighting function here is $1 / \chi^{2}$.

Fig. 6 shows the distribution of the absolute value of the similarity $\zeta$ obtained by equation (4) in each of the considered parameters. The $x$-axis indicates the distribution of the relative error for each considered parameter, while the $y$-axis is the statistical frequency in percentage. Zero in the similarity $\zeta$ means that the considered parameters of the simulation match with the solution. In each panel of Fig. 6, we plot the distribution of $\overline{\operatorname{Par}}_{i}$ Mod (black) and the distribution of the relative error obtained by the lowest $\chi^{2}$ solution (green). At the top right of each panel the percentage of simulations with a relative error $\leq 20$ per cent (defined here as a good match) is shown. We note that for 10 parameters considered in the simulations shown in Fig. 6, excluding the magnetic field ( $\sim 41$ per cent) and loop asymmetry ( $\sim 43$ per cent), the plots show a high percentage of good matches. On the other hand, although we added two new geometric parameters (loop inclination and loop asymmetry) we note that they are not easy to fit.

Although the lowest $\chi^{2}$ solution shows a small advantage in relation to the weighted mean, both methods result in quite similar distributions. We note that this difference is not sufficient to choose the best solution because there is a degeneracy of solutions in the neighbourhood of the closest solution. Thus, we decided to compute the weighted mean due to its robustness in relation to a single value (lowest $\chi^{2}$ solution only).

Table 2 shows two examples (simulations picked at random) with solutions A (lowest $\chi^{2}$ and weighted mean solutions) and $\mathrm{B}$ (weighted mean solution only). The bold numbers indicate the input parameters of the searched model (simulation). The number 1 in the first column of the simulation A indicates the lowest $\chi^{2}$ solution. The numbers $1-10$ in the first row of the table are parameter index only (see Table 1). The mean is the weighted mean of the parameters for the hundreds best models. ${ }^{3}$

Fig. 7 shows the lowest $\chi^{2}$ solution in the data base for simulation A referred to in Table 2. The overlaid contour plots in each image are at levels 30 and 50 per cent of image brightness maximum. The 10 analysed parameters of the simulation and solution are also shown in Table 2.

Although the images and spectra differ slightly, we note that many of the parameters are recovered and show errors in the acceptable range considered earlier (relative error $\leq 20$ per cent) in this section (see the parameters in Table 2 for comparison). The relative error as defined by equation (4) in percentage of each parameter ( $i$ ) for both possible solutions, the lowest $\chi^{2}$ and the averaged $\overline{\operatorname{Par}}_{i}$ Mod are as follow (the relative error for the averaged in parenthesis): $\epsilon: 18.5$ per cent (23.9 per cent), $\delta: 11.4$ per cent ( 0.84 per cent), $N_{\text {nth }}: 0.0$ per cent (2.0 per cent), $q: 32.8$ per cent (20.7 per cent), $A z: 1.6$ per cent (1.2 per cent), $R_{\text {arc }}: 27.9$ per cent (20.0 per cent), $F_{\text {sep }}: 12.3$ per cent (2.6 per cent), $H_{\text {arc }}: 9.5$ per cent (11.1 per cent), $t: 11.1$ per cent (49.7 per cent), and $B: 27.9$ per cent (3.7 per cent).

Thus, in simulation A the error in matching the lowest $\chi^{2}$ solution is on average around 15.3 per cent, while the weighted mean is around 13.6 per cent. Furthermore, the differences in the parameters might be understood in terms of the complexity of the dependence of gyrosynchrotron emission on so many parameters, including the effects of radiative transfer.

Within this simulation A (mean solution), it is seen that important parameters for the analysis of the emitting source, magnetic field, the number density of non-thermal electrons and spectral index of non-thermal electrons, are clearly recovered. On the other hand, the parameters of the source geometry play an important role in characterizing the source. These differences in the source sizes (see

\footnotetext{
${ }^{3}$ One hundred models ordered by increasing $\chi^{2}$.
} 

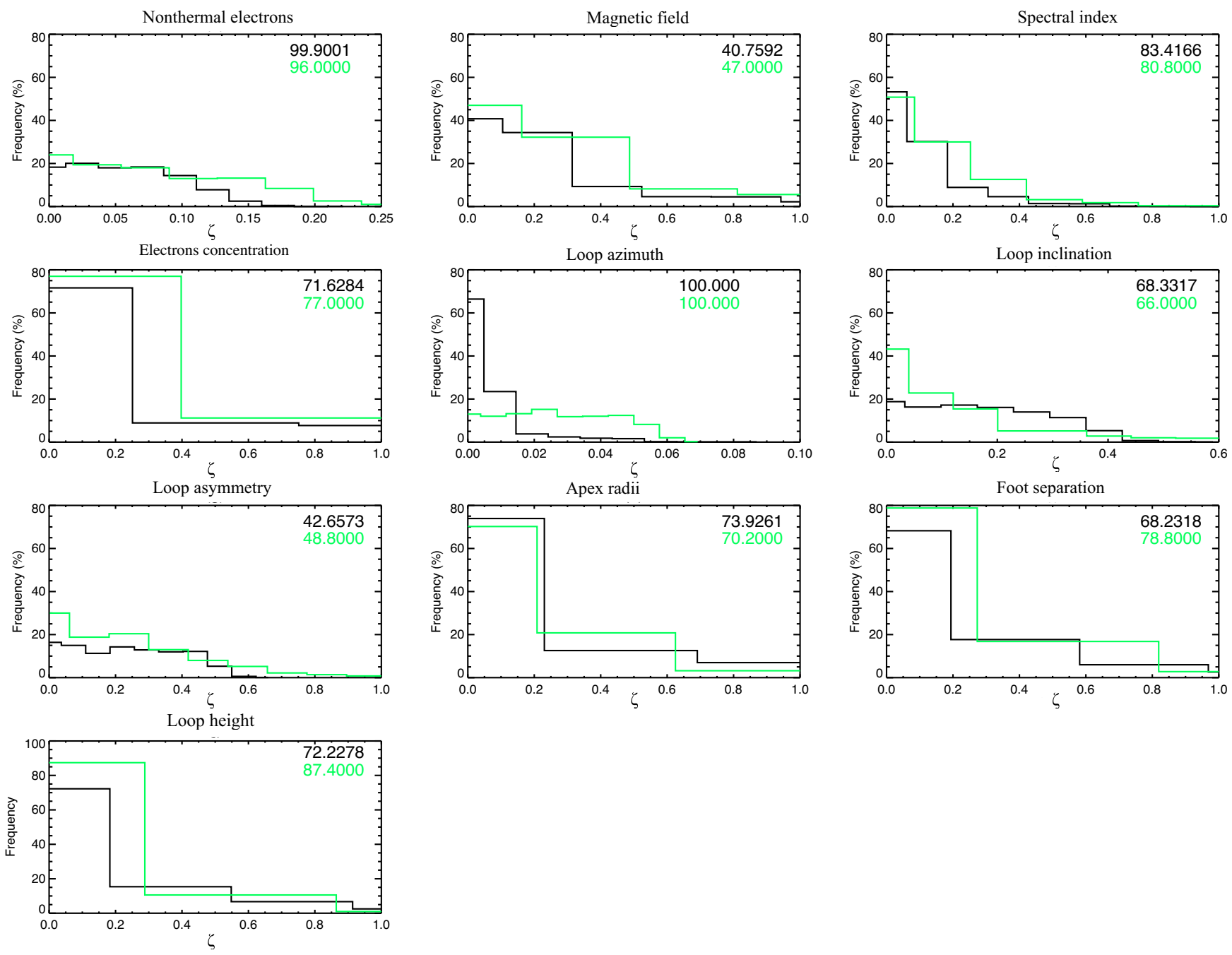

Figure 6. Distribution of errors in each parameter for the search simulations. The $x$-axis represents the distribution of relative error in each parameter. The $y$-axis is the percentage of the statistical frequency. Errors of the lowest $\chi^{2}$ solution are plotted as a green colour and the black colour is the weighted mean. Top right numbers are the percentage of elements with an error $\leq 20$ per cent.
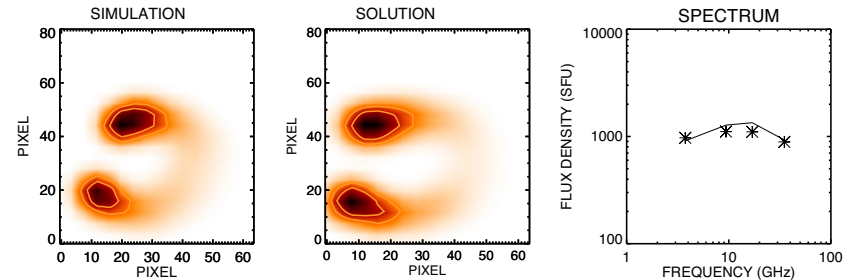

Figure 7. The left-hand panel of the figure shows the simulation, the middle panel shows the solution with the lowest $\chi^{2}$, and the right-hand panel shows the simulated spectrum denoted with asterisks and the solution with a continuous line.

columns 6,7 , and 8 in Table 2 , that refer to the radius of the loop apex, foot separation, and loop height, respectively) mainly affect the image and the shape of the spectrum as discussed in the literature (e.g. Stahli, Gary \& Hurford 1989). Therefore, by recognizing the high degeneracy of solutions, we prefer to choose the weighted mean solutions, as mentioned. Moreover, by saving the set of parameters used for this mean solution, it is possible to refine the fit using this set as an initial guess in different Monte Carlo methods constraining the ranges.

Fig. 8 shows images obtained with the weighted mean parameters $\left(\overline{\mathrm{Par}}_{i}^{\mathrm{Mod}}\right.$ ) of simulation B (also see Table 2). The top row of Fig. 8 shows the images at four frequencies $(3.75,9.4,17$, and $34 \mathrm{GHz})$ of simulation B. The bottom row shows the calculated images using the weighted mean parameters. The spectra are shown in Fig. 9. The asterisks are the spectrum of the simulation $\mathrm{B}$, and the continuous line is the calculated spectrum.

We noted earlier that some refinement can be made to improve the spectrum fitting by calculating new parameters around the suggestions given by the data base, as we will show later in Section 7.

We conclude that the results obtained so far are an improvement for flare analysis using a forward-fitting method by giving a good initial match or at least a shorter range of parameter space for further improvement.

Furthermore, we analysed the distribution of the relative errors among the parameters (also, see Fig. 6) in our sample of searched simulations. It is noted that the majority of the parameters have er- 

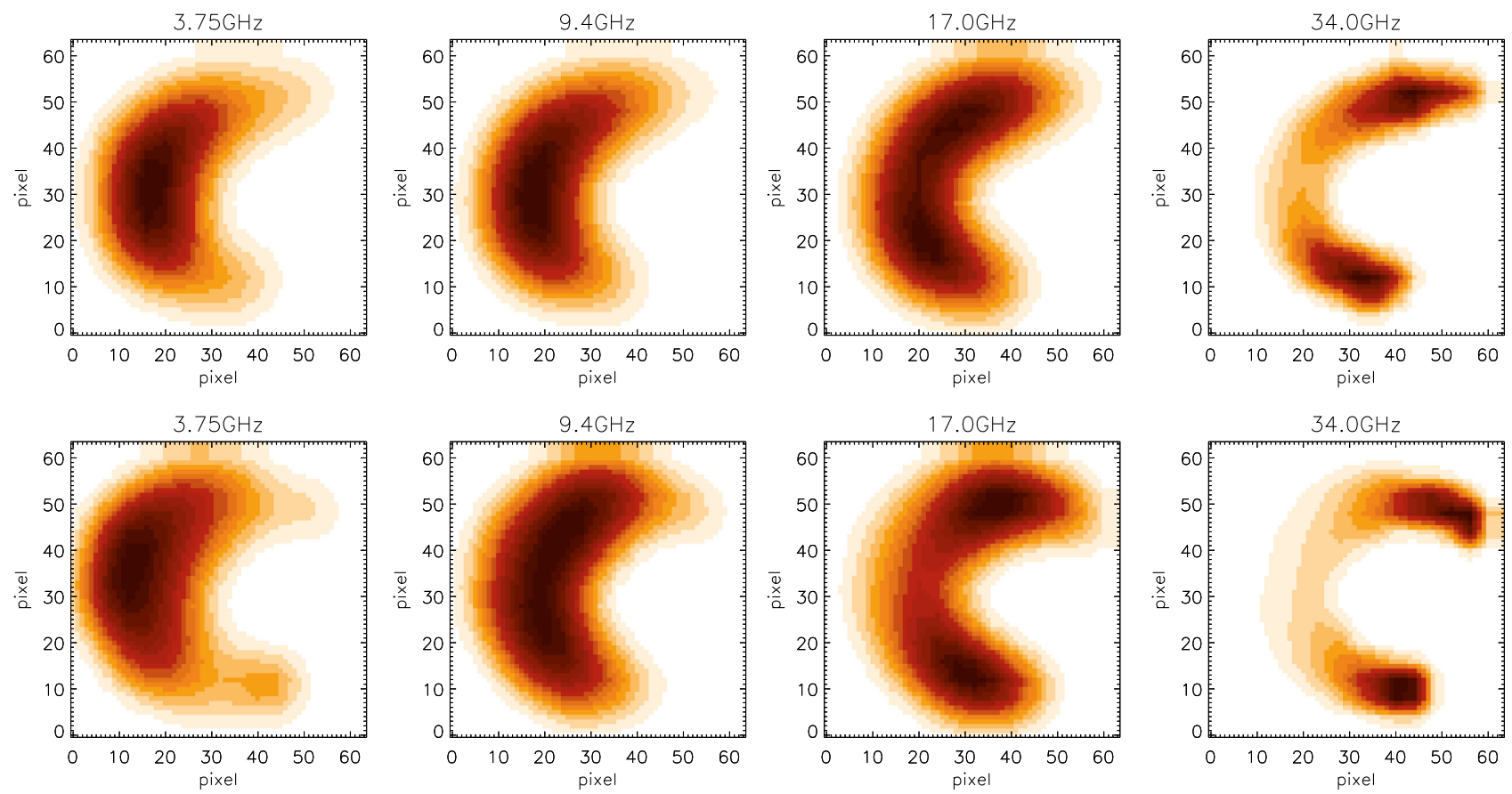

Figure 8. Top row shows the calculated images at four frequencies of the simulations shown in simulation B (Table 2) and the bottom row shows the calculated images of the solutions with the weighted mean parameters.

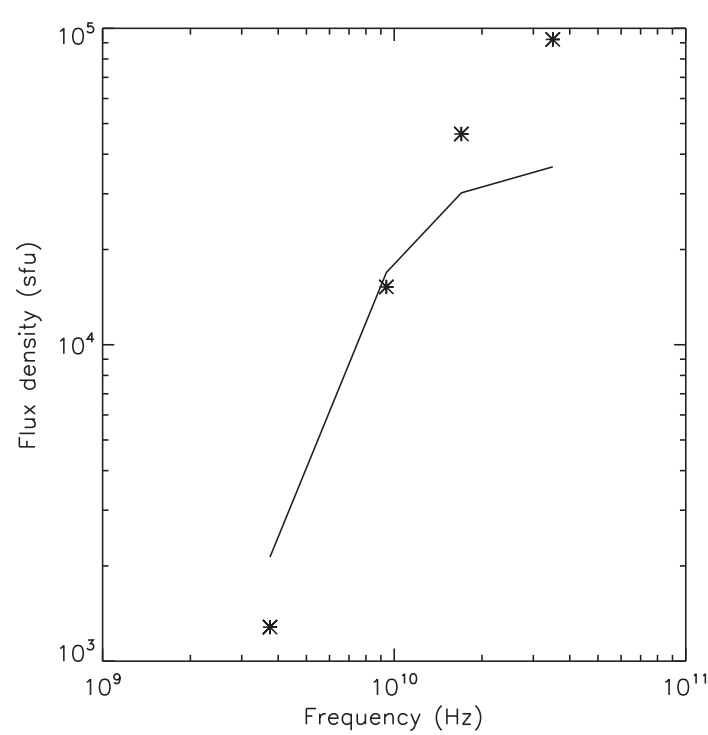

Figure 9. The asterisks in this figure show the spectrum of simulation $B$ from Table 2, and, the continuous line, the spectrum of the fitting of the solution.

rors less than 20 per cent on average, with the exception of the loop asymmetry and magnetic field which show a lack of models in the acceptable range of solution. To make improvements, one possibility is to generate an initial parent population from the data base best matches to run a genetic algorithm (e.g. Gibson \& Charbonneau 1996). Also, some refinements can be made, if we estimate some parameters from the observations, as we will show later when we apply the data base to the 2002 May 31 flare.

To finalize, we may say that the use of known information in the data base such as azimuth, inclination, or asymmetry which may be obtained via magnetogram or multiwavelength observations, improves the chances of success. Knowing the azimuth to an accuracy of $10 \mathrm{deg}$, the percentage of $\sum_{\mathrm{i}=1}^{10}\left|\zeta_{i}\right| \leq 2$ (on average $\zeta_{\mathrm{i}} \sim 0.2$ for all parameters) is 60 per cent. This number increases to 64 per cent when both inclination and asymmetry are known, as obtained in 1000 searched simulations.

\section{STATISTICS OF NORH FLARES INFERRED WITH THE CURRENT DATA BASE}

\subsection{The geometry bias}

As we saw in Fig. 4, the data base of models covers the whole range of observed fluxes by the NoRP observations with a very broad spread of points over the plotted range. However, from our view, one of the most important results is the relationship between the flux density at $17 \mathrm{GHz}$ (typically in the optically thin regime) and the geometrical property revealed by the emitting area (see the grey dots in Fig. 10). This helps us to discard the models that are not important for the Sun in our data base, thus avoiding the contribution of the parameters resulting from unlikely flares.

In Fig. 10, it is possible to see a correlation between the flux density and the emitting area (or source diameter, defined here as the square root of the emitting area). If we apply this correlation condition, we may find some important statistical properties of the parameters revealed by the observed solar flares.

The grey asterisks in Fig. 10 show plots from the NoRH flares (758) of effective diameter (selected from NoRH flare list) at $17 \mathrm{GHz}$ versus NoRP data flux. The black dots are the convolved (beam size 10 arcsec) models at $17 \mathrm{GHz}$. The right-hand panel of Fig. 10 shows the boundary range of the data removed from the data base.

We analysed what had been removed from the data base. The black bars in Figs 11-13 show the distribution of the model param- 

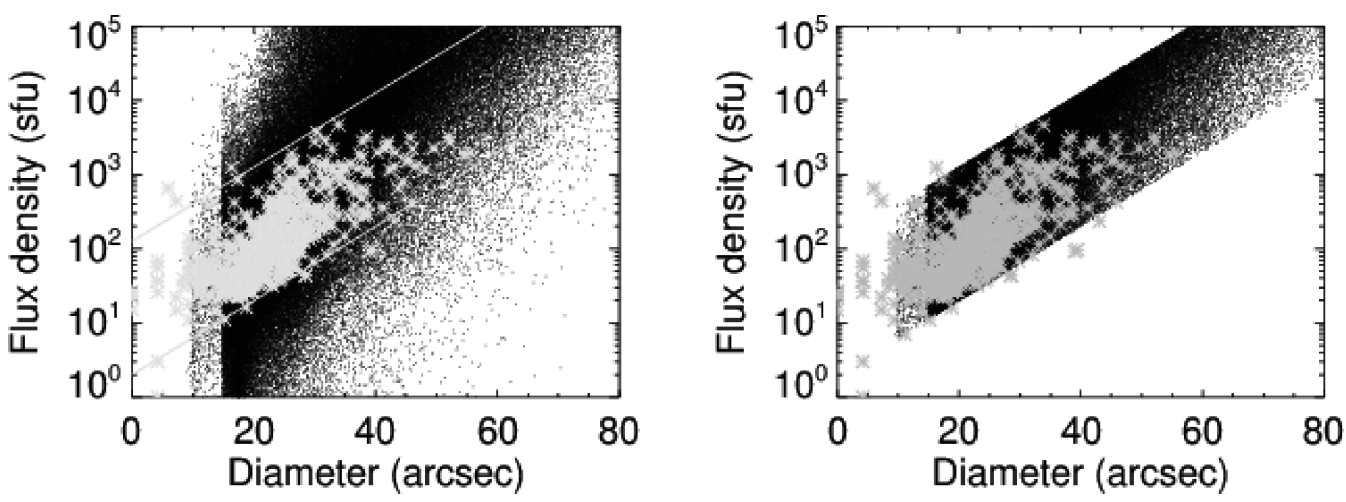

Figure 10. The black dots show the data base of $17 \mathrm{GHz}$ convolved models as in Fig. 4, and the grey asterisks show Nobeyama data at $17 \mathrm{GHz}$. The right-hand panel shows the same plot excluding the models at the upper and lower parts of the red boundary to fit the data for statistical analysis.

eters in the data base described above. They are almost homogeneously distributed (black bars) over the parameter range defined for this data base (see Table 1). Panel (j) in Fig. 12 shows a lack of models as a decision to maintain the loop structure, thus avoiding an apex radius greater than the loop height. Therefore, we set the loop height to be greater than the loop radius.

Fig. 11 shows the distribution of the energy parameters such as non-thermal electron number density (log scale), energy spectral index, and magnetic field of both the model data base without any modification (black bars), and the model data base after excluding the range of non-observed flares described in Fig. 10 (grey bars). We note a tendency of inferred characteristics of NoRH flares to be populated with the number density of non-thermal electrons tending to the lower end of the range $\sim 10^{6} \mathrm{~cm}^{-3}$ (panel a of Fig. 11), although the centre of the data base distribution is $10^{7} \mathrm{~cm}^{-3}$. This may imply in reduction of the lower limit of this parameter in the model data base to improve future diagnostics. We also note in panel (b) that the photospheric magnetic field has preferred values above $1800 \mathrm{G}$. Such values of the magnetic field as high as $1800 \mathrm{G}$ have been reported for photospheric and low coronal regions (e.g. Akhmedov et al. 1982; White, Kundu \& Gopalswamy 1991; Shibasaki et al. 1994). On the other hand, the spectral indices present a maximum peak around 3 (Panel c). This result is in accordance with the statistic $(\bar{\delta}=2.7, \sigma=0.8)$ obtained in Section 3 from the observational data.

We also show in panel (d) of Fig. 11 the location of the nonthermal electrons at the time of the burst maximum. It is noted that these flares have a tendency to be homogeneous or loop-top dominated where $\epsilon$ ranges from $\sim 0.8$ to 1.6 and then the histogram drops drastically. This drop means that we found the upper limit of $\epsilon$ from the observations at $17 \mathrm{GHz}(\epsilon<1.8)$.

In Fig. 12, black bars denote geometric parameter histogram plots such as loop-radius, loop-height, and foot-separation of models in the data base while grey bars show the same excluding unlikely flares. From these parameters, we note that NoRH flares have a tendency to populate sizes greater than $0.04 \mathrm{R}_{\odot}\left(\sim 2.6 \times 10^{9} \mathrm{~cm}\right)$ for loop height and loop feet separation, as well as for sizes greater than $0.015 \mathrm{R}_{\odot}\left(\sim 10^{9} \mathrm{~cm}\right)$ for the apex radius.

We also show in Fig. 13 histogram plots of the loop orientation, such as loop-azimuth and loop-inclination, both for models with (black) and excluding unlikely NoRH flares (grey), as in previous plots. Panel (g) of Fig. 13 shows the asymmetry of the magnetic field. There appears to be no major variations between these parameters.

\section{APPLICATION TO THE 20020531_000725 UT FLARE}

We carried out one example of the implementation of the data base with 3D models of an M2.4 GOES class flare observed on the East limb of the Sun on 2002 May 31 (Fig. 15). It is one of the many wellanalysed events from the work of Shih, Lin \& Smith (2009), and it clearly shows a loop-like geometry. This M2.4 flare was located at S30E85. The maximum peak in $9.4 \mathrm{GHz}$ at NoRH was observed at 00:07:25 UT. Kawate et al. (2012) made a joint analysis from hard $\mathrm{X}$-ray and microwave data of this event as well.

We collected the four fluxes in the frequencies ranges in the data base on the NoRP website without any additional calibration, and both images at 17 and $34 \mathrm{GHz}$ at NoRH. NoRP works at seven discrete frequency channels, namely $1,2,3.75,9.4,17,34$, and $80 \mathrm{GHz}$, with a $0.1 \mathrm{~s}$ time resolution. Our main goal here is to use the approach described in Section 4 to find the best model which fits the observed NoRP spectrum and NoRH images at 17 and $34 \mathrm{GHz}$.

From the brightness distribution map, NoRH $17 \mathrm{GHz}$ image does not show, in many cases, a clear geometry of a loop-like flare. Eventually, this happens either due to loop-top emission or small-size loops that are not resolved by the instrument. Therefore, for magnetic field orientation, we end up aligning brightness distributions of two oval shapes. We note that in some cases we may use a magnetogram and EUV images to help to identify the loop azimuth and magnetic field asymmetry.

From the magnetogram, we found that the maximum positive and negative B is $\sim 2200 \mathrm{G}$ for this 2002 May 31 flare; which suggests low asymmetry. The inferred parameters of the observation (computed weighted mean) from our data base search are shown in Table 3. Furthermore, Table 3 also shows the refined parameters (solution) obtained after calculating new models not included in the data base by using a genetic algorithm known as Pikaia (Charbonneau 1995) to improve the image and spectrum matches. Although the weighted mean azimuth shows positive orientation, it differs greatly from the solution. Azimuth is hard to measure when observing near the limb. It is difficult to decide about the alignment of the loop foot-points in relation to the local parallel.

From our analyses, we found a microwave loop-top emission that is quite clear from the image (Fig. 15) of this 2002 May 31 flare near the limb. This effect might be explained by an enhancement of energetic electrons at the top of the loop occurring as a result of a transverse pitch angle anisotropy caused by the accelerating mechanism as suggested in the work of Melnikov et al. (2002). 
Table 3. Best data base representation.

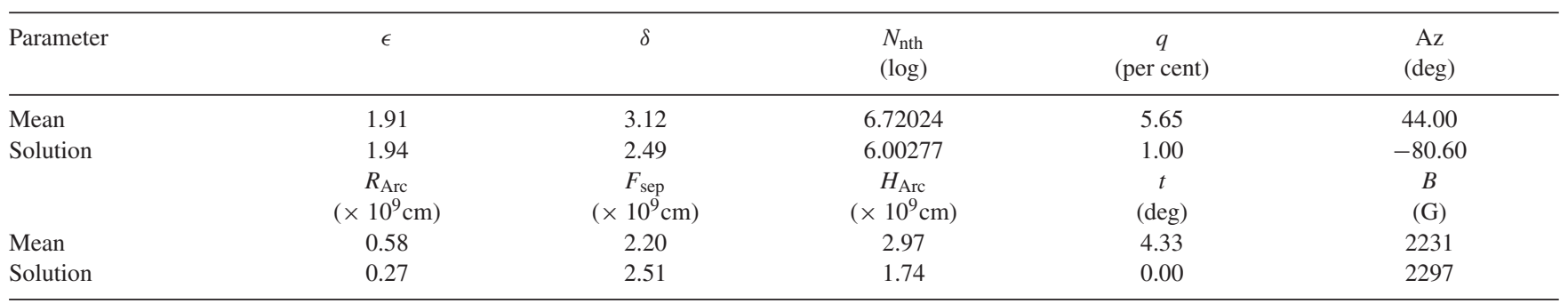

Fig. 14 shows plots of the spectra. The asterisks show the observed spectrum obtained from NoRP website, the continuous line shows our refined solution. We did not find a good solution in the data base to fit the observations without any refinement. In some cases, the image appeared to be inconclusive or the spectrum did not match. We note that the data base is still moderate in size and the percentage of good fits may be measured in terms of the statistics of the parameters, as discussed in Section 5. Therefore, we chose the weighted mean of the hundred best models in the data base as a guess input for a final refinement with the Pikaia genetic algorithm.

Fig. 15 shows the observed NoRH maps at 34 and $17 \mathrm{GHz}$ on a red scale. The maps are overlaid with white contours at 50 per cent of NoRH image brightness maximum and black contour plots of the model for comparison. It is clearly seen that the contours do not perfectly match, but they reproduce the shape of the observed loop. This may imply that many of the geometric parameters (see Table 3 ) of the model are roughly in the same range as the observable.

Although the plots of the distribution of each parameter mentioned above in the model are available in the data base, they are not shown here.

\section{DISCUSSION}

This data base was constructed considering radio emission produced by electrons spiralling on the magnetic field lines in a dipolar magnetic field. From the literature (e.g. Svestka 1981; Bastian et al. 1998), it is assumed that in the radio range the emission comes from the non-thermal electrons that are accelerated in the corona and then propagate down to the foot-points of the loops with a commonly accepted power-law distribution in energy. Nevertheless, when taking into account the comparison between radio and hard X-ray observations, our 3D magnetic loop radio modelling agrees reasonably well with the literature in relation to the location and sizes of the radio source. The ambient plasma is supposed to be a quiescent background with some temperature and density profiles. It is widely known that the radio emission emanates from the chromosphere to corona where the electrons are trapped due to magnetic mirroring. Thus, the number density of non-thermal electrons refers to different locations in the loop and different distribution of trapped or precipitating particles, from which it follows that a detailed determination of the emitting region parameters in microwaves and hard $\mathrm{X}$-rays is important.

We present an alternative approach to the time-consuming process to calculate a non-homogeneous model - each time one is needed for a flare analysis - by providing a data base of precalculated models constrained by some statistical properties of a large sample of Nobeyama observations. We know that this data base is still limited in terms of the wide range of parameter combinations needed to calculate the gyrosynchrotron emission to describe all possible scenarios of flares in the microwave. We statistically analysed 10 important parameters, that in general cannot be directly observed, to explain a flare as shown in Fig. 6. We found that 8 out of 10 parameters in the simulations were recovered with a relative error $\leq 20$ per cent on average. We consider this result an improvement for flare analysis in terms of the time consumed with the calculations.

Although the data base was produced using equally distributed parameters from the lower to upper limits, the geometric bias discussed in Section 6.1 results in differences in the distributions of geometry, magnetic field, and energy parameters. In the energy parameters, the observed solar bursts show preferences for lower non-thermal electron density and a slight preference for higher densities for the limits used here. The magnetic field distribution shows some preference for higher values than the centre of the data base range we used. We also noted some shift in the distribution for looptop events. In general, the geometric parameter distribution shows a tendency for bigger loops.

Differences between individual solutions are mainly due to the degeneracy of results with quite similar $\chi^{2}$ in the dominion of 10 parameters. This is the reason that we preferred the averaged solution weighted by the $\chi^{2}$.

Table 3 shows the best parameters inferred from the M2.4 GOES class flare observation. We can see that the data base provides a mean solution with good agreement with the observations, but the calculated image and spectrum using the set of parameters still do not match the observations. However, the refinement resulted in a final solution which better agreed with the observations.

Fig. 14 shows the best-fitting spectrum obtained from our analysis with a good match. The spectrum of the event has the typical shape of the non-thermal gyrosynchrotron emission mechanism (e.g. Gary 1989), with a peak around $9.4 \mathrm{GHz}$. Thus, we assume 17 and $34 \mathrm{GHz}$ in the optically thin regime to calculate the spectral index (according to Dulk \& Marsh 1982) which results in $\delta=2.12$. We note that the $\delta$ found here is from a single power law and may be considered as an average of the indices in a multipower-law spectrum. When comparing the fitted parameters in this paper, such as electron spectral index, with those obtained by Kawate et al. (2012; their Table 2) using a triple power law (Table 3), one may consider that our result is between their range of power-law 2 and 3, and agrees with a typical value in the statistical analysis carried out by Silva, Wang \& Gary (2000).

Fig. 15 shows in colour the loop shape of NoRH $17 \mathrm{GHz}$ images overlaid with the black contours of our solution convolved with the typical beam size of NoRH. The result of this first analysis is in good agreement with the observations. It is evident that this data base covers the entire range of fluxes on the NoRH website. We are still working on this search engine for use in future analyses. 

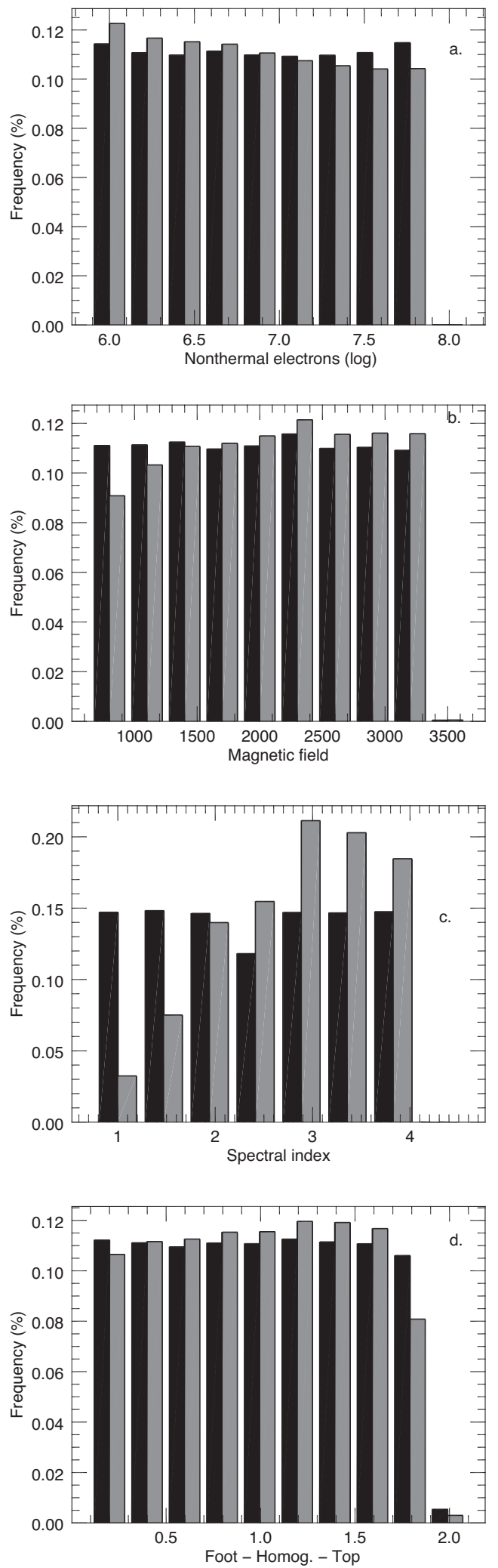

Figure 11. Histogram plots of the energy parameter distribution implemented in the data base. The $x$-axis is the energy parameters, and the $y$-axis is the count rate of the elements. The black bars are the data base of energy parameters without any modification, while the grey bars are the same parameters after excluding the range of NoRH observed flares.
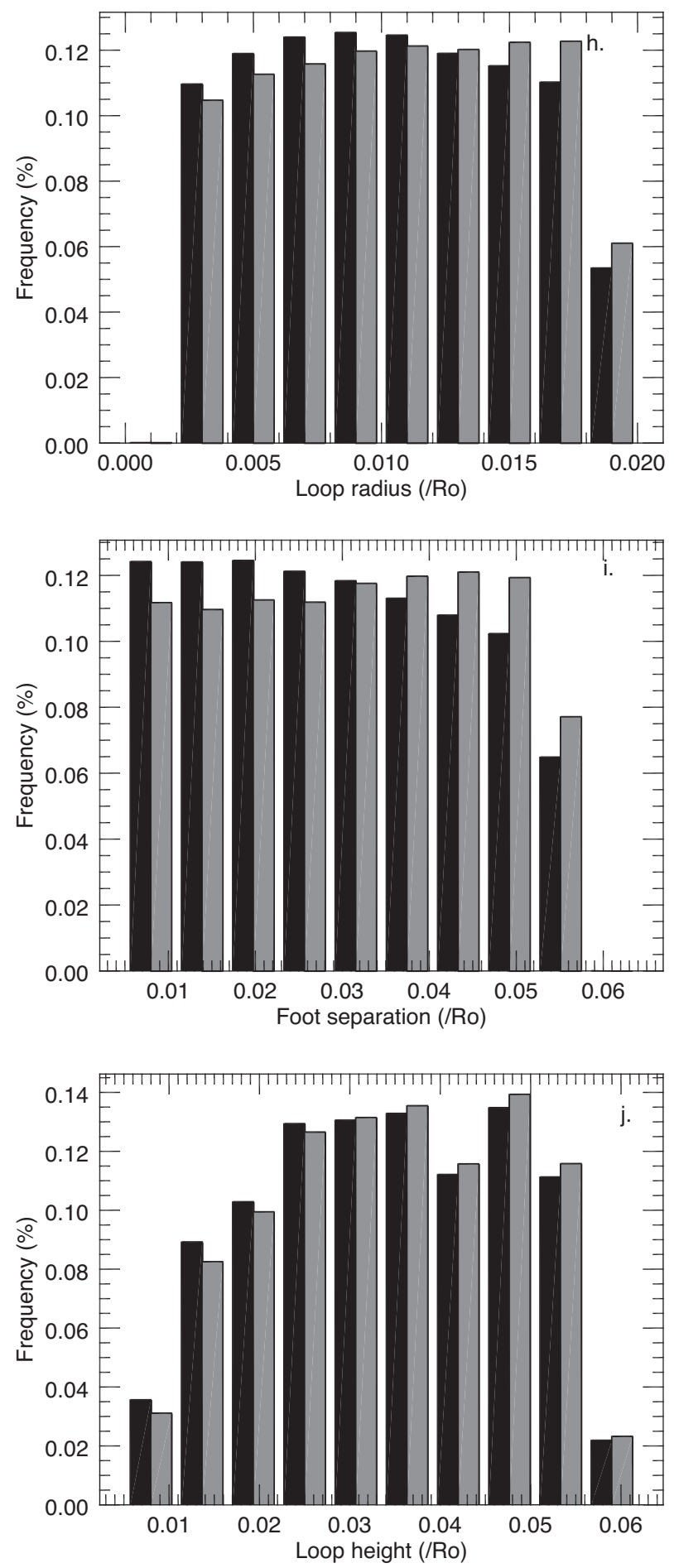

Figure 12. Histogram plots of geometric parameter distribution such as loop-radius, loop-height, and foot-separation implemented in the data base. The $x$-axis is the parameters and the $y$-axis is the count rate of elements. The black colour shows the data base model parameters and the grey bar is the same after excluding the range of NoRH observed flares.

\section{CONCLUSIONS AND FUTURE STUDIES}

The aim of this study is to present an alternative approach for the analysis of flares using a recently developed data base of 3D loop models. The data base was presented initially in PAPER 1 with some limitations in the geometry, ranges of parameters to calculate 

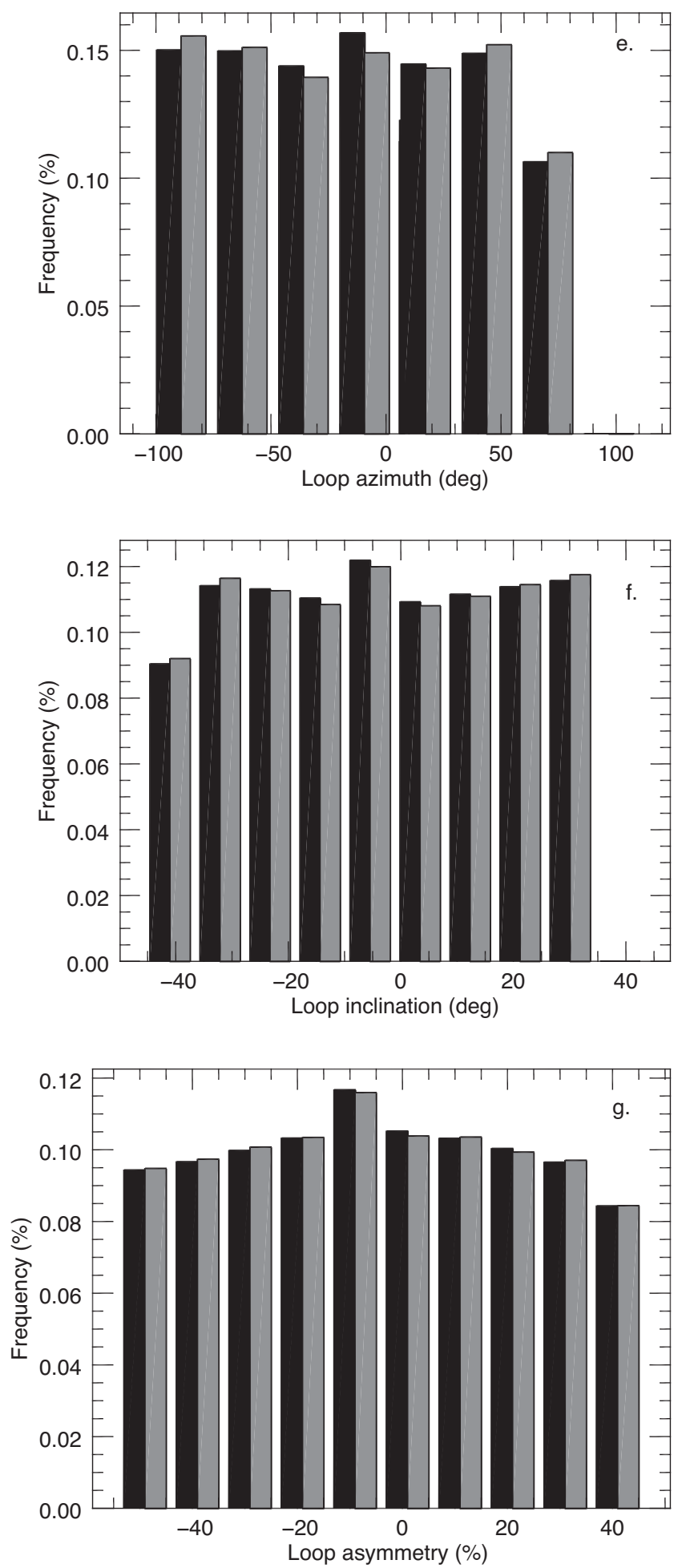

Figure 13. Histogram plots of the geometric parameter distribution such as loop-inclination and loop-azimuth implemented in the data base. Panel (g) shows the asymmetry of the magnetic field. The $x$-axis is the parameters and the $y$-axis is the count rate of elements.

the gyrosynchrotron emission, and a small number of calculated models. The actual data base was constructed with 17 variable parameters in an effort to reproduce the flare characteristics. We understand that a high number of variables result in a large number of combinations and consequently in an extremely large universe

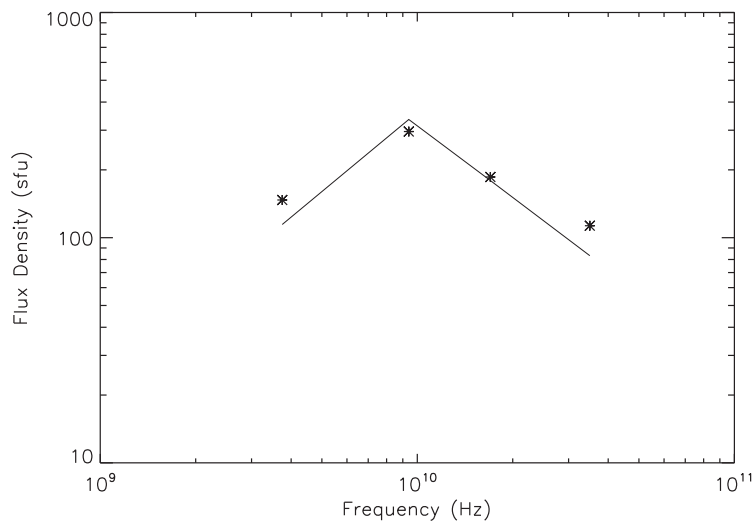

Figure 14. Plots of the calculated and observed spectra. The asterisks are the observed NoRP spectrum; the thick line is the fit.
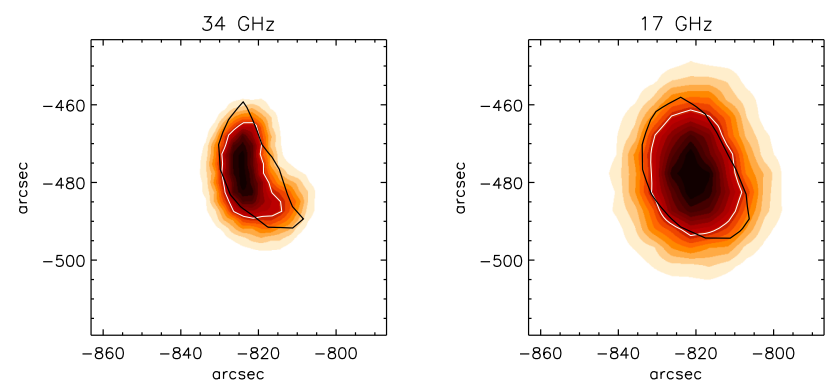

Figure 15. Images of NoRH 34 and $17 \mathrm{GHz}$ (red scale) brightness temperature with contours overlaid (white) at 50 per cent of image maximum and model (black) at 50 per cent.

of solutions which are difficult to characterize with a small sample of models. Also, the side effect of the number of variables involved is a data base with a high degeneracy of solutions. Our results show some advantages regarding the reduction of the time of flare analysis, providing an initial guess of parameters and confining the space of possible solutions.

The implementation of 3D loop models based on dipolar geometry is an improvement from homogeneous flare models in terms of the flare source description. Thus, the considered analysis with the loop-like geometry should allow for better inference than the homogeneous approach. In addition, this data base speeds up the search for the best geometrical representation of the observed brightness temperature of NoRH maps and the best spectral fit of NoRP, by using a reasonably large volume of pre-constructed models with different scenarios.

The relevant results found in Section 5 reveal that 80 per cent of all 10 parameters, except loop asymmetry and the magnetic field, used to fit the simulations were fitted with good agreement (relative error $\leq 20$ per cent) which reinforces the use of this approach. We noted that the probability of success in the simulations increased with the data base growth.

We also noted in Section 6 some statistical results by the preferred range of physical parameters of NoRH flares, such as $\delta \sim 3$, $N_{\text {th }} \lesssim 10^{7} \mathrm{~cm}^{-3}, B \gtrsim 2000 \mathrm{G}$, and the geometry that results in bigger loops with height $\underset{\approx}{\approx} 2.6 \times 10^{9} \mathrm{~cm}$ and feet separation $\gtrsim 10^{9} \mathrm{~cm}$. We also conclude that NoRH flares at $17 \mathrm{GHz}$ have some preference for loop-top events. For this particular data base, further work could be carried out to fine-tune and improve the results obtained so far.

The analysis of the M2.4 GOES class flare presented produced an acceptable solution when compared with the literature (Kawate 
et al. 2012). We consider the result of the 2002 May 31 flare only a moderate success, as we did not find a reasonable fit for both the spectrum and images at 17 and $34 \mathrm{GHz}$ in the current data base. We had to use the models found as an initial family of members for a genetic algorithm to improve the result. This is an indication that this data base still needs to be expanded while we try to develop a better way to fine-tune the resultant model with some optimizing routine exploring the near parameter space in the data base.

\section{ACKNOWLEDGEMENTS}

We would like to thank the SKA SA for all their support and the Brazilian agency CAPES for providing financial support for one of the authors (V.A.C). We would also like to extend our thanks to Professor Donald Kurtz for proofreading this paper and to the Nobeyama Radioheliograph, which is operated by NAOJ/Nobeyama Solar Radio Observatory. Finally, we are grateful to FAPESP for the partial financial support through the grant 2014/11156-4 for the computing infrastructure.

\section{REFERENCES}

Akhmedov S. B., Gelfreikh G. B., Bogod V. M., Korzhavin A. N., 1982, Sol. Phys., 79, 41

Alissandrakis C., Preka-Papadema P., 1984, A\&A, 139, 507

Bastian T. S. Benz, a. O., Gary D. E., 1998, A\&A, 36, 131

Charbonneau P., 1995, ApJS, 101, 309

Costa J., Simões P., Pinto T., Melnikov V., 2013, PASJ, 65, 5

Dulk G., Marsh K., 1982, ApJ, 259, 350

Fleishman G. D., Nita G. M., Gary D. E., 2009, ApJ, 698, 183

Fleishman G. D., Nita G. M., Gary D. E., 2011, Proc. IAU Symp., 274, 280

Fletcher L. et al., 2011, Space Sci. Rev., 159

Gary D. E., 1985, ApJ, 297, 799

Gary G., 1989, ApJ, 69, 323

Gibson S., Charbonneau P., 1996, BAAS, 28, 76
Hurford G. J., Read R. B., Zirin 1984, Sol. Phys., 413

Kawate T., Nishizuka N., Oi A., Ohyama M., Nakajima H., 2012, ApJ, 747, 131

Klein K., 1987, A\&A, 183, 341

Kucera T. A., Dulk G. A., Kiplinger A. L., Winglee R. M., Bastian T.S., Graeter M., 1993, ApJ, 412, 853

Kundu M., Nindos A., White S., Grechnev V., 2001, ApJ, 557, 880

Kuznetsov A. A., Kontar E. P., 2015, Sol. Phys., 290, 79

Lee J. W., Gary D. E., Zirin H., 1994, Sol. Phys., 152, 409

Lee J., Nita G. M., Gary D. E., 2009, ApJ, 696, 274

Liu K., Wang Y., Zhang J., Cheng X., Liu R., Shen C., 2015, ApJ, 802, 35

Melnikov V. F., Reznikova V. E., Yokoyama T., Shibasaki K., 2002, in Wilson A., ed., ESA SP-506: Solar Variability: From Core to Outer Frontiers., ESA, Noordwijk, p. 339

Melrose D. B., Brown J. C., 1976, MNRAS, 176, 15

Nakajima H., et al., 1994, IEEE Proceedings, 82, 705

Nindos A., White S., Kundu M., Gary D., 2000, ApJ, 533, 1053

Nita G., Gary D., Lee J., 2004, ApJ, 605, 528

Nita G. M., Fleishman G. D., Kuznetsov A. A., Kontar E. P., Gary D. E., 2015, ApJ, 799, 236

Petrosian V., 1981, ApJ, 251, 727

Ramaty R., 1969, ApJ, 158, 753

Shibasaki K., et al., 1994, PASJ, 46, L17

Shih A. Y., Lin R. P., Smith D. M., 2009, ApJ, 698, L152

Silva A., Wang H., Gary D., 2000, ApJ, 545, 1116

Simões P. J. A., 2009, PhD thesis, Instituto Nacional de Pesquisas Espaciais (INPE), São José dos Campos

Simões P., Costa J., 2006, A\&A, 453, 729

Simões P., Costa J., 2010, Sol. Phys., 266, 109

Stahli M., Gary D., Hurford G., 1989, Sol. Phys., 120, 351

Svestka Z., 1981, in Priest E.R., ed., Flare observations, Solar Flare Magnetohydrodynamics. Gordon \& Breach, New York, p. 47

White S. M., Kundu M. R., Gopalswamy N., 1991, ApJ, 366, L43

White S., et al., 2011, Space Sci. Rev., 159, 225

This paper has been typeset from a $\mathrm{T}_{\mathrm{E}} \mathrm{X} / \mathrm{L} \mathrm{T} \mathrm{E} \mathrm{X}$ file prepared by the author. 